\title{
A top-down, three-scale numerical analysis of wafer-to-wafer metallic bonding
}

\author{
Aldo Ghisi ${ }^{1}{ }^{1}$, Stefano Mariani ${ }^{1}$, Alberto Corigliano ${ }^{1}$ and Giorgio Allegato ${ }^{2}$ \\ 1 Politecnico di Milano, Dipartimento di Ingegneria Civile e Ambientale, Piazza L. da Vinci 32, 20133 \\ Milano, Italy; E-Mails: aldo.ghisi@polimi.it (A.G.); stefano.mariani@polimi.it (S.M.); \\ alberto.corigliano@polimi.it (A.C.)
}

2 STMicroelectronics, AMS - MEMS Technology Development, Via C. Olivetti 2, 20041 Agrate Brianza, Italy; E-Mail: giorgio.allegato@st.com (G.A.)

* Author to whom correspondence should be addressed; E-Mail: aldo.ghisi@ polimi.it (A.G.);

Tel.: +39-02-2399-4310; Fax: +39-02-2399-4300.

Published: 1 June 2014

\begin{abstract}
To study the sensitivity to micro-scale imperfections of the strength of a metallic, wafer-to-wafer MEMS bonding, we propose a three-scale numerical (finite element) approach. At the wafer level (macro-scale), accounting for the whole metallic sealing through nonlinear springs connecting the two silicon wafers modelled as thin plates, we link the force transferred by each single MEMS die to the external pressure applied to the wafers. This force is next used as an index for the input pressure at the die level (meso-scale), where the geometry of the metallic rings is accurately described: the local stress field at the interface between the upper and lower metallic rings is so obtained. Finally, a local (micro-scale) model is used to link the aforementioned local stress field in each die to the bonding strength: representative volumes of the rings getting into contact, accounting in a statistically way for the relevant surface roughness (which is on the order or tens of nanometers at most), are adopted to obtain the relationship between the external pressure and the percentage of sealed area. This information is exploited to assess the properties of the rings, in terms of expected bonding strength.
\end{abstract}

Keywords: MEMS; wafer bonding; multi-scale analysis; nonlinear mechanics. 


\section{Introduction}

Wafer-to-wafer thermo-compressive bonding [1] is a valuable option to accomplish the sealing of a MEMS cavity. Its economic advantage derives from the possibility of operating on the whole wafer. However, the process currently shows some technological difficulties: if (unleaded) metals are chosen as a sealing material, then thin films have to be deposited on the two facing wafers, and temperature and load histories must be carefully designed in order to obtain effective bonding. The combined effects of pressure and thermal loading lead to the re-crystallization of the metals; hence, the mechanical properties of the bonding evolve from the original ones and, in general, they are rather difficult to estimate at the micro-scale [2]. The nature of the surface roughness, the orientation of the crystal grains are only some of the variables concurring to define the quality of the bond [3], and they are difficultly linked with the global control parameters of the process, like temperature and pressure inside the bonding chamber.

In this work (see also [4]) we describe a multi-scale computational procedure, based on finite element mechanical analyses, to estimate the bonding strength starting from the aforementioned extensive parameters, and provide a link between quantities at the macro-scale (i.e. process parameters) and at the micro-scale.

\section{Three-scale approach}

We identify three length-scales to address the analysis of the thermo-compression bonding. At the top level, labelled macro-scale, Figure 1(a), the whole 8-inches wafers or a big portion of them (like the quarter shown in the picture) are considered: the two silicon wafers, modelled as plates, are pushed against each other by the external (i.e. chamber) pressure. The interface in between the two plates, i.e. the pattern of the metal sealing rings, is modelled through nonlinear springs: each spring is placed at the center of mass of the relevant metallic ring for each MEMS die, and through its force-elongation relation it accounts in a simplified way for the mechanical link in the out-of-plane direction. The adopted load-elongation law for a single spring is depicted in Figure 2; possible differences in the ring thickness and area are respectively represented by a spreading of the initial gap $g$ and of the stiffness $k=E A / l, E$ being the Young's modulus of the metal constituting the ring, $A$ the ring in-plane area and $l$ the total ring thickness. The obtained force on the single spring is the output of the macro-scale analysis, to be transferred in a proper way as input pressure at the meso-scale, where only the corresponding die is accounted for, see Figure 1(b).

The portions of the upper and lower silicon wafers modelled at the meso-scale and connected through the metallic rings, are handled as three-dimensional solids. In the analysis, a perfect or hard contact condition is assumed to hold between die and cap or, more specifically, between the two surfaces of the rings connected to die and cap and getting in touch. The pressure field at the interface between the two rings is retrieved as the main output at this length-scale. This quantitative information is next used as the input for the analysis at the micro-scale.

Figure 1. (a) Model of one quarter of a silicon wafer (macro-scale); (b) model of die substrate, cap and metal rings (meso-scale); (c) representative volume of the two metal rings entering into contact (micro-scale). 


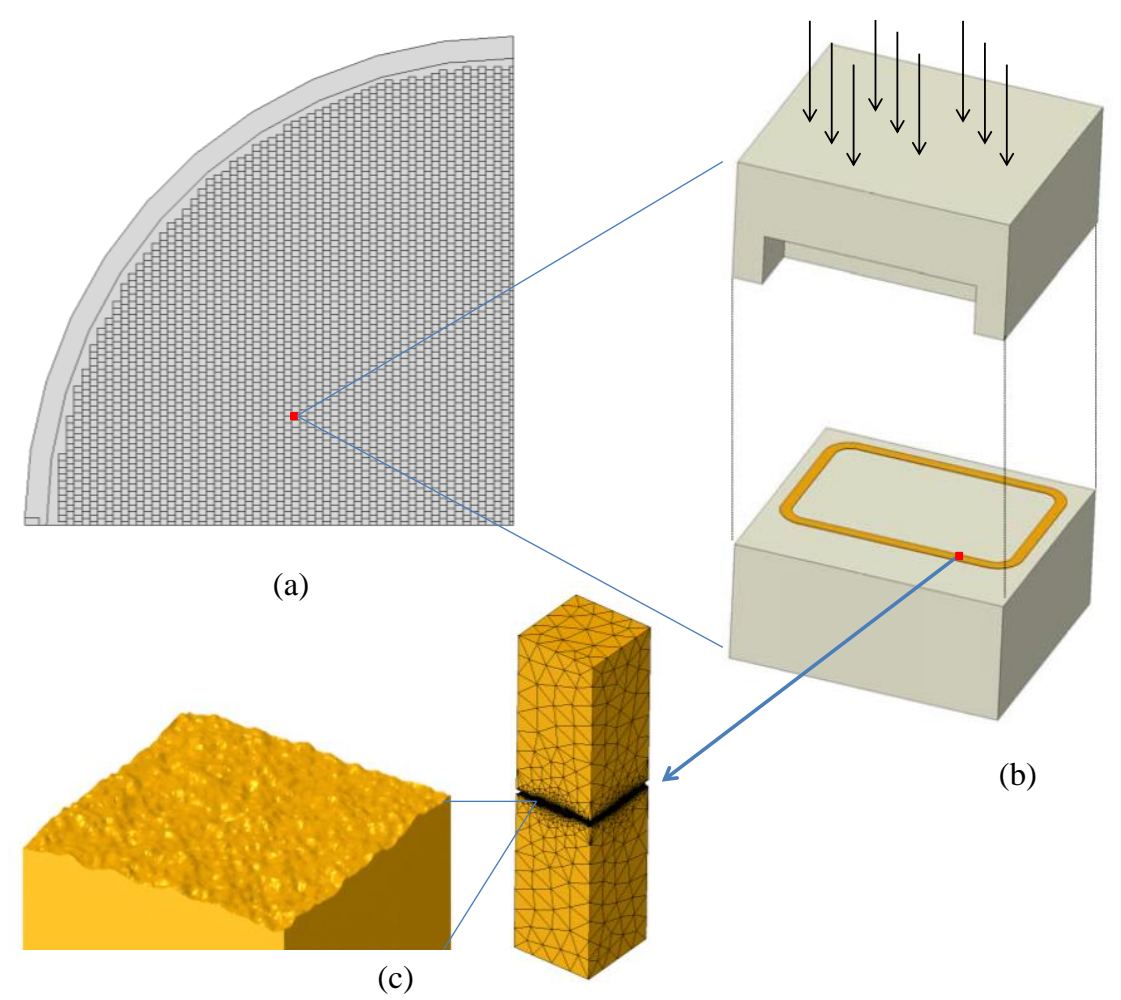

Figure 2. Nonlinear spring force-elongation model for the sealing rings at the macro-scale.

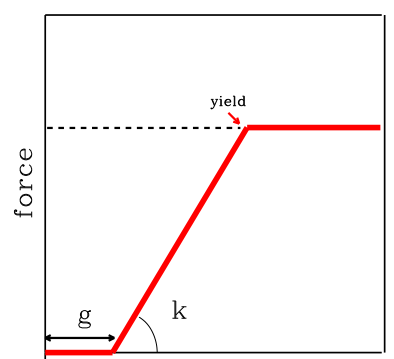

displacement

Table 1. Reference mechanical properties.

\begin{tabular}{lcc}
\hline Material property & Units & Value \\
\hline Si Young's modulus [100] & $\mathrm{MPa}$ & 130,000 \\
Si Poisson's ratio & - & 0.28 \\
Si shear modulus & $\mathrm{MPa}$ & 80,000 \\
Metal Young's modulus & $\mathrm{MPa}$ & 75,000 \\
Metal Poisson's ratio & - & 0.4 \\
Metal yield strength & $\mathrm{MPa}$ & 50 \\
Metal hardening modulus & $\mathrm{MPa}$ & 1 \\
\hline
\end{tabular}

Finally, at the micro-scale the contact surfaces of the rings are artificially reconstructed (see [5-6]) for a representative volume of the metal sealing rings, see Figure 1(c). The relationship between the pressure and the actual surface area entering into contact is adopted to provide an estimate of the bond strength. This procedure allows, in principle, to quantitatively evaluate the bonding effectiveness on the whole wafer as a 
function of extensive quantities like the temperature and pressure in the bonding chamber, and also of local features like e.g. the roughness of the ring surfaces.

A purely mechanical, finite element analysis has been carried out. The relevant properties of the silicon and of the metal constituting the sealing rings at the bonding temperature are collected in Table 1. Silicon has been assumed to be elastic (as tensile stresses never attain its characteristic strength) and orthotropic, while the rings have been assumed to be elastic-plastic with a very small hardening modulus.

\section{Results and Discussion}

An overview of the stress pattern over the wafer, as arising from the macro-scale analysis, is shown in Figure 3. The overall external force corresponding to this solution is $38 \mathrm{kN}$, applied by the hydraulic jack on the top (cap) wafer in the bonding chamber. The maximum principal stress field in the silicon wafers is obviously influenced by the stiffness of each single spring representing a sealing ring: in the reported solution, every ring has been assumed to feature a thickness equal to $4 \pm 0.2 \mu \mathrm{m}$. The offset from the thickness mean value has been randomly assigned to the springs, in compliance with a uniform probability distribution: as already reported, this scattering enters into play both for the initial gap and for the elastic spring stiffness. It results that there is no sensitivity to this geometry scattering, as the stress pattern is shown to be highly repetitive.

Figure 3. Macro-scale analysis: stress pattern (in MPa) on the silicon wafer.

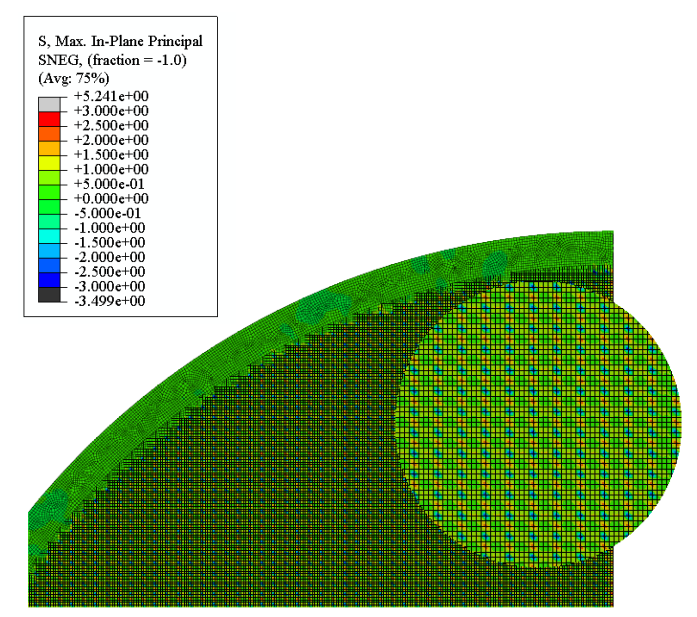

At the meso-scale, the effects of the pressure acting on small portions of the metal ring interface are evaluated. A vertical cross-section of the stress field, as depicted in Figure 4(a), shows that the highest stress concentrations occur at the connection between the rings, where re-entrant corners are present. This happens as the widths of the upper and lower rings are different, in order to allow for in-plane misalignments during the sealing process. At the center of the ring, the out-of-plane stress flattens out: this is the most critical sealing region, where a check on whether the external pressure is sufficient to assure a good bonding is necessary. The pressure acting along the longitudinal plane of the ring slightly varies along the axis, as shown in Figure 4(b). It obviously depends also on possible in-plane misalignments, which can shift the relative position of the upper and lower rings. As an example, in Figure 4(c) the effects are reported for a misalignment along the horizontal direction (according to the view shown in same Figure 4(b)). 
Figure 4. Meso-scale analysis: (a) out-of-plane stress field (in $\mathrm{MPa}$ ) in a model cross-section; (b) normal pressure acting on the interface ring; (c) variation of the pressure across the width of the interface ring, due to misalignment.
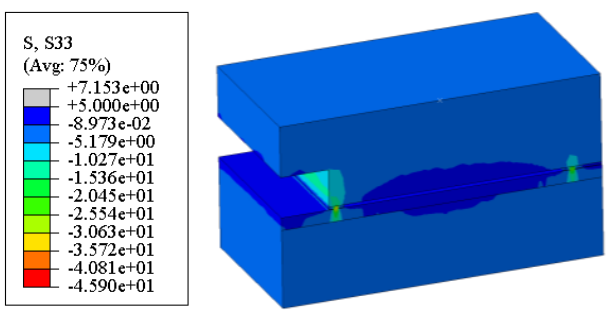

(a)

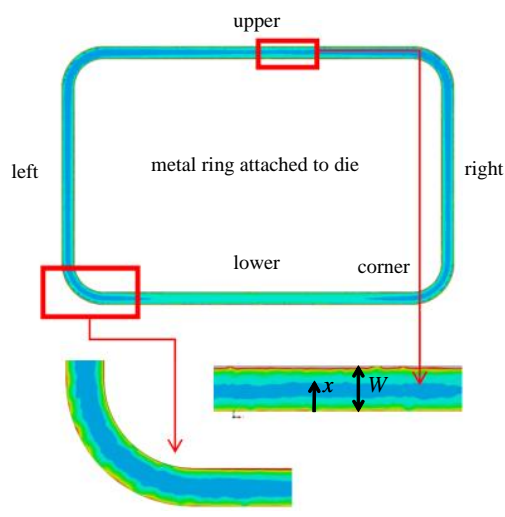

(b)

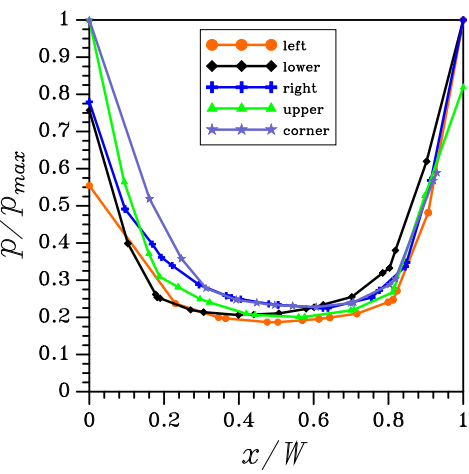

(c)

Figure 5. Micro-scale analysis: (a) $\mathrm{rms}=10 \mathrm{~nm}$, area into contact (red) at an applied pressure equal to $0.0004 \mathrm{GPa}$; (b) $\mathrm{rms}=10 \mathrm{~nm}$, area into contact (red) at an applied pressure equal to $0.605 \mathrm{GPa}$; (c) contact area vs pressure relationship, at varying surface roughness.

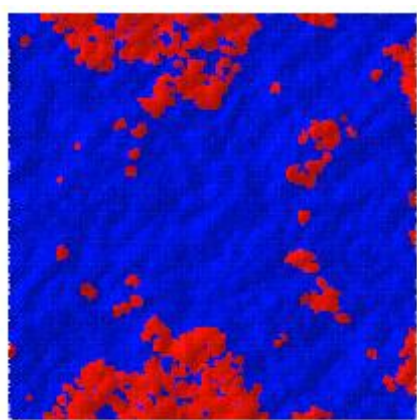

(a)

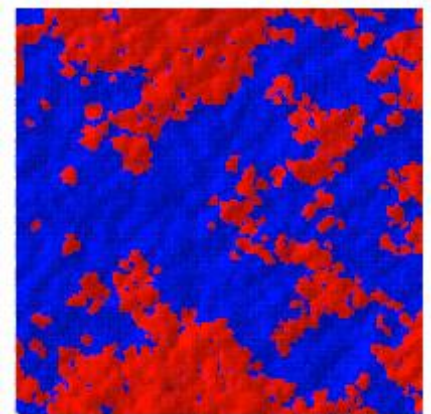

(b)

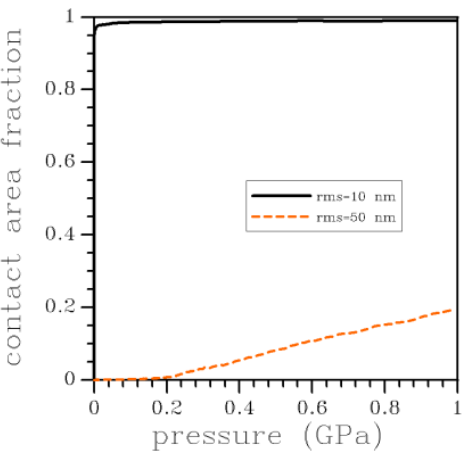

(c)

At the micro-scale a representative volume of the rings, see Figure 1 (c), whose dimensions are $1 \cdot 1 \cdot 4$ $\mu \mathrm{m}^{3}$, has been adopted. The surface roughness has been digitally built [5-6] according to a Gaussian density probability function with a specified standard deviation from a flattened plane, or root mean square (rms). Two different rms values, $10 \mathrm{~nm}$ for a low roughness case and $50 \mathrm{~nm}$ for a high roughness case, have been considered. When the upper ring is pushed against the lower one by the pressure coming from the mesoscale analysis, the surface peaks on both sides are flattened due to the elastic-plastic deformation, and the overall force transmitted through the contacted area increases. The area getting into contact is depicted in red in Figures 5(a) and 5(b) for two different pressure values and for the low roughness case; Figure 5(c) shows the corresponding contact area vs pressure relationship for both the rms values considered. This allows an evaluation of the percentage of the area in contact as a function of the out-of-plane pressure, and provides an estimate of the bonding strength. 


\section{Conclusions}

A numerical procedure, able to provide an evaluation of the strength of a wafer-to-wafer thermocompression bonding, has been described. The approach is based on a three-scale formulation from the wafer, macro-scale level down to the ring, micro-scale level, accounting for the surface roughness of the metal rings themselves. The intermediate meso-scale analysis at the single die level, allows dishomogeneities of the pressure acting at the interface between the two sealing rings to be evidenced.

The approach can link extensive variables, like the external force applied to the silicon wafer, and very local variables, like the surface roughness, to provide an estimate of the bonding strength. Statistical investigations of the effects of geometry imperfections, such as variations of the ring thickness, in-plane ring misalignments, and surface roughness characteristics, will be accounted for in future studies.

\section{Acknowledgments}

This work has been carried out within the framework of the Eniac Joint Undertaking Project Lab4MEMS, grant n. 325622. Partial financial support by STMicroelectronics through the Material Reliability project is also gratefully acknowledged.

\section{References}

1. Tsau, C.H.; Spearing, S.M.; Schmidt, M.A. Characterization of wafer-level thermocompression bonds. Journal of Microelectromechanical Systems 2004, 13, 963-971.

2. Greer, J.; Hosson, J.T.M.D. Plasticity in small-sized metallic systems: intrinsic versus extrinsic size effect. Progress in Material Science 2011, 56, 654-725.

3. Gui, C.; Elwenspoek, M.; Tas, N.; Gardeniers, J. The effect of surface roughness 263 on direct wafer bonding. Journal of Applied Physics 1999, 85, 7448-7454.

4. Ghisi, A.; Corigliano, A.; Mariani, S.; Allegato, G. A Multi-Scale Approach to Wafer to Wafer Metallic Bonding in MEMS. 15 $5^{\text {th }}$ IEEE International Conference on Thermal, Mechanical and Multi-Physics Simulation and Experiments in Microelectronics and Microsystems, EuroSimE 2013, Wroclaw, April 15-17.

5. Ardito, R.; Corigliano, A.; Frangi, A. Modelling of spontaneous adhesion phenomena in Micro-ElectroMechanical Systems. European Journal of Mechanics - A/Solids 2013, 39, 144-152.

6. Hu, Y.Z.; Tonder, K. Simulation of 3-D random surface by 2-D digital filter and Fourier analysis. International Journal of Machine Tools and Manufacture 1992, 32, 83-90.

(C) 2014 by the authors; licensee MDPI, Basel, Switzerland. This article is an open access article distributed under the terms and conditions of the Creative Commons Attribution license (http://creativecommons.org/licenses/by/3.0/). 